\begin{tabular}{|c|c|c|c|c|c|}
\hline & $\begin{array}{l}\text { All Patients } \\
(n=353)\end{array}$ & $\begin{array}{c}\text { Remission } \\
\text { CDAl }</=2.8 \\
(n=3)\end{array}$ & $\begin{array}{c}\text { LDA } \\
2.8<C D A \mid</=10 \\
(n=13)\end{array}$ & $\begin{array}{c}M D A \\
10<C D A \mid</=22 \\
(n=78)\end{array}$ & $\begin{array}{c}\text { HDA } \\
\text { CDAl>22 } \\
(n=259)\end{array}$ \\
\hline P29-Physical Function & $37.5 \pm 6.4$ & $50.1 \pm 6.07$ & $43.5 \pm 7.37$ & $39.7 \pm 6.83$ & $36.4 \pm 5.66^{*}$ \\
\hline P29-Anxiety & $54.5 \pm 10.3$ & $44.8 \pm 7.74$ & $50.4 \pm 9.12$ & $51.6 \pm 10.0$ & $55.7 \pm 10.24^{*}$ \\
\hline P29-Depression & $52.7 \pm 10.1$ & $43.7 \pm 4.62$ & $46.2 \pm 7.25$ & $49.8 \pm 10.18$ & $54.0 \pm 9.91^{*}$ \\
\hline P29-Fatigue & $59.9 \pm 9.6$ & $41.8 \pm 7.53$ & $51.1 \pm 9.57$ & $56.9 \pm 9.55$ & $61.5 \pm 9.04^{*}$ \\
\hline P29-Sleep Disturbance & $56.0 \pm 8.3$ & $52.8 \pm 14.36$ & $52.3 \pm 6.41$ & $54.1 \pm 8.48$ & $56.8 \pm 8.17$ \\
\hline $\begin{array}{l}\text { P-29 Ability to participate in } \\
\text { Social Roles and Activities }\end{array}$ & $42.3 \pm 8.2$ & $56.2 \pm 9.18$ & $49.9 \pm 8.43$ & $44.5 \pm 8.84$ & $41.2 \pm 7.50^{*}$ \\
\hline P29-Pain Interference & $64.0 \pm 7.5$ & $45.7 \pm 7.1$ & $54.3 \pm 7.81$ & $61.0 \pm 8.10$ & $65.5 \pm 6.35^{*}$ \\
\hline Fatigue SF 7a & $60.3 \pm 8.3$ & $43.0 \pm 10.19$ & $54.3 \pm 7.65$ & $57.5 \pm 7.22$ & $61.7 \pm 8.02^{*}$ \\
\hline Pain Interference SF 6b & $63.0 \pm 7.7$ & $43.5 \pm 4.33$ & $54.6 \pm 9.29$ & $59.9 \pm 8.03$ & $64.5 \pm 6.62^{*}$ \\
\hline $\begin{array}{l}\text { P29 Pain Intensity } \\
\text { (scored 0-10 scale) }\end{array}$ & $6.2 \pm 2.2$ & $2.0 \pm 3.46$ & $3.5 \pm 1.71$ & $5.4 \pm 2.32$ & $6.6 \pm 1.91^{*}$ \\
\hline
\end{tabular}

Conclusions: These interim data further support the viability of using PROMIS questionnaires to evaluate RA pts, and indicate in this predominantly HDA population of RA pts correlations between PROMIS and CDAl disease activity category. Confirmation of the baseline interim analysis findings with the fully enrolled AWARE study, as well as inclusion of longitudinal and subset analyses based on disease activity levels, will further define the role of PROMIS relative to CDAl in RA patients in a real world setting.

Disclosure of Interest: J. Curtis Consultant for: Janssen, AbbVie, Roche/Genentech, BMS, UCB, Myriad, Lilly, Amgen, Pfizer, Corrona, S. Kafka Employee of: Janssen Scientific Affairs, LLC, D. Parenti Employee of: Janssen Scientific Affairs, LLC, S. Black Employee of: Janssen Scientific Affairs, LLC, S. Xu Employee of: Janssen Research \& Development, LLC, Y. Wang Employee of: Janssen Research \& Development, LLC, C. Bingham III Grant/research support from: Janssen, PCORI, NIH, Pfizer, Consultant for: Janssen, AbbVie, Amgen, BMS, Celgene, Genentech/Roche, Lilly, Macrogenics, Meoblast, Novartis, NovoNordisk, Pfizer, Regeneron, UCB DOI: 10.1136/annrheumdis-2017-eular.5225

\section{THU0673 THE EUROPEAN CONSENSUS FINDING STUDY GROUP (ECFSG) HELPS CHARACTERIZING NEW TENTATIVE REFERENCE STANDARDS FOR AUTOANTIBODY MEASUREMENT}

J. Rönnelid $^{1}$, C. Dahle ${ }^{2}$, M. Blüthner ${ }^{3}$, E. Feist ${ }^{4}$, C. Dolman ${ }^{5}$, S.J. Thorpe ${ }^{5}$, E. Monogioudi ${ }^{6}$, I. Zegers ${ }^{6}$, P.L. Meroni ${ }^{7}$, D. Hamann ${ }^{8}$ on behalf of ECFSG. ${ }^{1}$ Department of Immunology, Genetics and Pathology, Uppsala University, Uppsala; ${ }^{2}$ Department of Clinical Immunology and Transfusion Medicine, Linköping University Hospital, Linköping, Sweden; ${ }^{3}$ MVZ Laboratory PD Dr. Volkmann \& colleagues, Department of autoimmune diagnostics, Karlsruhe; ${ }^{4}$ Department of Rheumatology and Clinical Immunology, Charité-Universitätsmedizin, Berlin, Germany; ${ }^{5}$ National Institute for Biological Standards and Control, Biotherapeutics Group, Potters Bar, Herts, United Kingdom; ${ }^{6}$ Joint Research Centre, European Commission, Geel, Belgium; ${ }^{7}$ Department of Clinical Sciences \& Community Health, University of Milan, Milan, Italy; ${ }^{8}$ Department of Immunopathology and Blood Coagulation, Sanquin Diagnostic Services, Amsterdam, Netherlands

Background: Since 1988, the European Consensus Finding Study Group on autoantibodies in rheumatic diseases (ECFSG), also known as the EULAR autoantibody study group, has been distributing sera with unspecified antibodies to European laboratories (presently $n=43$ ) for evaluation of different autoantibody measurement techniques in a clinical context. Use of reference materials helps to align test results by adopting internationally used measurement units, but reference materials are missing for many autoantibody specificities.

Objectives: Recently the scope for ECFSG was expanded to also include unbiased autoantibody characterization of serum/plasma specimens planned to constitute raw material for production of future autoantibody reference materials. Methods: Four samples were included to be evaluated as future tentative international reference materials for four different autoantibody specificities: double stranded/native DNA (dsDNA, evaluated in 2013/14), IgG anti-b2GP1, proteinase 3 (PR3) and myeloperoxidase (MPO, evaluated in 2015/16). The samples were included "blind", and evaluated broadly for multiple autoantibody specificities by participating laboratories.

Results: All or almost all participating laboratories detected the target specificities, and all four samples showed restricted autoantibody specificities related to the target specificity. Anti-dsDNA was detected by all laboratories using Crithidia luciliae, ELISA/EIA, FARR assay or ALBIA and all labs reported a homogenous ANA pattern. Other specificities were restricted to histones, nucleosomes and anti-Ku. All laboratories but one detected IgG anti-b2GP1 and IgG anti-cardiolipin, mostly in high levels, in the tentative IgG anti-b2GP1 reference standard, whereas corresponding IgA and IgM antibodies were absent. All laboratories detected anti-MPO, mostly monospecific and in high levels together with P-ANCA pattern in the anti-MPO reagent. Anti-PR3 and C-ANCA pattern, mostly in high levels/titers were detected by all laboratories in the tentative anti-PR3 reagent, irrespective of method used.

Conclusions: The expanded scope of ECFSG has enabled broad characterization of new tentative autoantibody reference standards. The anti-dsDNA specimen has been processed by the National Institute for Biological Standards and Control (NIBSC) for consideration as the 2nd WHO anti-dsDNA reference standard. The other materials are basis for certified reference material for IgG antimyeloperoxidase (ERM-DA476/IFCC), and the candidate reference materials for $\lg$ G anti-proteinase 3 (in certification) and for IgG anti-b2PG1 (in evaluation) from the Joint Research Centre.

Disclosure of Interest: None declared

DOI: 10.1136/annrheumdis-2017-eular.1743

\section{THU0674 ANTI-DFS70 ANTIBODY - A BIOMARKER THAT AID IN THE EXCLUSION OF ANA ASSOCIATED RHEUMATIC DISEASES}

K. Conrad $^{1}$, N. Röber ${ }^{1}$, M. Achtleitner ${ }^{1}$, M. Aringer ${ }^{2}$, S. Rudolph ${ }^{3}$, L. Unger ${ }^{4}$ A. Gräßler ${ }^{5}$, K. Lüthke ${ }^{6}$, M. Mahler ${ }^{7}{ }^{1}$ Medical Faculty of the TU Dresden, Institute of Immunology; ${ }^{2}$ University Hospital Carl Gustav Carus, Department of Internal Medicine and Rheumatology, Dresden; ${ }^{3}$ Admedia MVZ, Immune Center Chemnitz, Chemnitz; ${ }^{4}$ Municipal Hospital Dresden-Friedrichstadt, Department of Medicine I, Dresden; ${ }^{5}$ Medical Practice, Pirna; ${ }^{6}$ Medical Practice of Rheumatology, Dresden, Germany; ${ }^{7}$ Inova Diagnostics, San Diego, United States

Background: Positive ANA may lead to additional testing and potentially even inappropriate treatment in patients with rheumatic symptoms not caused by ANA associated rheumatic diseases (AARD).

Objectives: It has been shown that autoantibodies directed against lens epithelial derived growth factor (LEDGF), also named DFS70 according to the staining pattern (dense fine speckled) and molecular weight of the target antigen $(70 \mathrm{kDa})$, are common among ANA positive individuals with no evidence of AARD $[1,2]$. The aim of our study was to evaluate if autoantibodies directed against DFS70 can be used to exclude AARD in ANA positive patients.

Methods: Anti-DFS70 antibody were determined by chemoluminescence assay (CIA) in sera of 352 apparently healthy controls (AHI), 1048 patients of an ANA positive routine cohort, 579 patients with AARD (300 SLE, 76 idiopathic inflammatory myopathies, 167 systemic sclerosis, 36 Sjögren's syndrome), 56 patients with undifferentiated connective tissue disease (UCTD), and 660 nonAARD patients (302 rheumatoid arthritis, 94 ANCA-associated vasculitis, 87 atopic rhinitis, 135 pediatric patients with celiac disease, and 42 autoimmune liver diseases).

Results: In AHI and in the non-AARD cohort, anti-DFS70 antibodies occur with a prevalence of $5.1 \%$ and $2 \%$, respectively. Of the 1048 selected routine sera, $205(19.6 \%)$ were positive for anti-DFS70 antibodies. Up to now, clinical reports are available for 116 of anti-DFS70 positive patients in this group. The diagnoses were widely scattered (nonspecific rheumatic symptoms, arthritis, thyreoiditis, asthma, psoriasis, tumor, infections, inflammatory bowel disease), but no definite AARD could be diagnosed. In the AARD group, only 6 of 579 patients $(1.2 \%)$ were positive for anti-DFS70 antibodies, all of them also show disease specific autoantibodies (two anti-Scl70 antibody positive SSc, one anti-RNAPIII antibody positive SSc, one Ro-52 positive SSc, one anti-Mi-2 antibody positive IIM, one SLE patient with multiple autoantibodies including dsDNA antibodies). In patients with UCTD, $6(10.7 \%)$ were anti-DFS70 antibody positive in the absence of disease specific autoantibodies. Up to now, no development of an AARD was observed in these patients.

Conclusions: Anti-DFS70 antibodies are frequently observed in sera with chromatin binding antibodies in the absence of disease specific autoantibodies. If anti-DFS70 antibodies are positive in the absence of AARD specific autoantibodies, an AARD can be excluded with high certainty.

\section{References:}

[1] Dellavance A, Viana VS, Leon EP, Bonfa ES, Andrade LE, Leser PG. The clinical spectrum of antinuclear antibodies associated with the nuclear dense fine speckled immunofluorescence pattern. J Rheumatol 2005;32,2144-49.

[2] Mahler M, Hanly JG, Fritzler MJ. Importance of the dense fine speckled pattern on HEp-2 cells and anti-DFS70 antibodies for the diagnosis of systemic autoimmune diseases. Clin Dev Immunol 2012; Article ID 4943356.

Disclosure of Interest: None declared

DOI: 10.1136/annrheumdis-2017-eular.5915

\section{THU0675 DEVELOPMENT AND PSYCHOMETRIC VALIDATION OF A TOOL TO ASSESS THE FEARS OF PATIENTS WITH CHRONIC INFLAMMATORY RHEUMATIC DISEASES: THE FAIR SCALE}

L. Gossec ${ }^{1,2}$, P. Chauvin ${ }^{3}$, C. Hudry ${ }^{4}$, G. Cukierman ${ }^{5}$, V. Saulot ${ }^{6}$

F. Russo-Marie ${ }^{6}$, T. de Chalus ${ }^{5}$, J.M. Joubert ${ }^{5}$, A. Saraux ${ }^{7}$, F. Berenbaum ${ }^{8}$ ${ }^{1}$ UPMC Univ Paris 06: ${ }^{2}$ AP-HP. Hôpital Pitié Salpêtrière; ${ }^{3}$ INSERM, UPMC Univ Paris 06: ${ }^{4}$ AP-HP, Hôpital Cochin, Paris; ${ }^{5}$ UCB Pharma, Colombes; ${ }^{6}$ Arthritis Fondation Courtin, Neuilly-sur-Seine; ${ }^{7} \mathrm{CHU}$ la Cavale Blanche and Université de Bretagne Occidentale, Brest; ${ }^{8}$ UPMC Univ Paris 06, AP-HP, Hôpital Saint-Antoine, Paris, France

Background: Patients (pts) with chronic inflammatory rheumatic diseases (CIRDs) such as rheumatoid arthritis (RA) and axial spondyloarthritis (axSpA) have fears related to their disease that can negatively impact health-related quality of life and compromise treatment adherence.

Objectives: To develop and validate a patient-reported outcome (PRO) ques- 
tionnaire to explore fears related to CIRDs using the Fears Assessment in Inflammatory Rheumatic Diseases (FAIR) Scale.

Methods: The preliminary questionnaire included 44 items (23 related to fears) most frequently cited by pts in a qualitative study of 50 French pts. ${ }^{1}$ Each item was formulated as an affirmative sentence and scored from 0 (completely disagree) to 10 (totally agree). Item scores were summed to provide a total score. The questionnaire was finalised and validated. Pts diagnosed with RA (EULAR/ACR criteria) or axSpA (ASAS criteria), recruited during routine visits by 100 participating rheumatologists across France, completed the preliminary questionnaire, HAD (Hospital Anxiety and Depression) and $\mathrm{AHI}$ (Arthritis Helplessness Index) scores. Redundant items (inter-item correlation coefficient $>0.65$ ) were eliminated. For the others, internal consistency (Cronbach alpha) and the factorial structure of the scale (principal component analysis) were assessed. Pts were classified according to their level of fears (cluster analysis) and corresponding score thresholds were determined (ROC analysis). The final questionnaire was independently translated into English and back into French twice, with reconciliation of the translated texts.

Results: 672 pts were included: 432 RA pts (mean \pm SD disease duration was $13.1 \pm 11.4$ years, DAS28[ESR] was $2.6 \pm 1.2,77.3 \%$ were taking biologics) and 240 axSpA pts (disease duration was $13.8 \pm 10.6$ years, BASDAI was $3.3 \pm 2.2$, $72.7 \%$ were taking biologics). The final FAIR Scale included 10 questions (Table) with total scores ranging $0-100$. Mean $\pm S D$ scores were $51.2 \pm 25.4$ in RA and $60.5 \pm 22.9$ in axSpA. Three pt groups were identified, characterised by high, moderate and low level of fears $(17.2 \%, 41.1 \%$ and $41.7 \%$ of the population, respectively). The corresponding thresholds of the total score were 77 and 51 , respectively. Fear scores were correlated with HAD scores for anxiety $(r=0.47)$ and depression $(r=0.40)$ and with the $\mathrm{AHI}(r=0.50)$.

Table: FAIR Scale - 10 items [a]

\section{Fears about the progression and consequences of the disease:}

\section{B-7: I am afraid of suffering like I did in the past.}

D-3: I am afraid that my disease is rapidly getting worse.

D-5: I am afraid that my spinal column or some of my bones will fuse.

D-7: I am afraid of not having anyone to help me if I lose my autonomy.

E-1: I am afraid that I will no longer be able to carry out my daily activities.

E-5: I am afraid that people will think of me as disabled.

C-6: I am afraid that at some point there will be no more effective treatments for my disease.

Fears about treatments:

C-2: I am afraid of the side effects of treatments for my disease.

C-3: I am afraid that the treatments of my disease may cause cancer.

C-4: I am afraid that the treatments of my disease will become less effective.

[a] Each item is scored between 0 (strongly disagree) and 10 (strongly agree); total score of 100.

Conclusions: The FAIR Scale is a 10-question PRO to evaluate disease-related fears in CIRD pts. In this pt population, $17.2 \%$ had high fear scores, contrasting with a disease that is often well-controlled. The FAIR Scale was associated with psychological distress. This psychometrically-validated and easy to use questionnaire could be used to improve pt-physician dialogue in CIRDs and also be of value in clinical studies. Further validation in other populations is needed.

References:

[1] Berenbaum F. PloS One 2014; 9(12):e114350.

Acknowledgements: The authors acknowledge Costello Medical Consulting, funded by UCB Pharma, for editorial assistance. This study was funded by UCB Pharma and Arthritis Foundation Olivier Courtin.

Disclosure of Interest: L. Gossec Grant/research support from: UCB Pharma, Lilly, Consultant for: AbbVie, BMS, Celgene, Janssen, Novartis, MSD, UCB, P. Chauvin: None declared, C. Hudry: None declared, G. Cukierman Employee of: UCB Pharma, V. Saulot: None declared, F. Russo-Marie: None declared, T. de Chalus Employee of: UCB Pharma, J. M. Joubert Employee of: UCB Pharma, A. Saraux Consultant for: UCB Pharma, F. Berenbaum: None declared DOI: 10.1136/annrheumdis-2017-eular.1282

\section{THU0676 ANTI-RO/SS-A 52-KDA ANTIBODIES: A MARKER FOR LUNG FIBROSIS IN RHEUMATIC DISEASES}

L. Garcia Montoya, C.N. Sáenz-Tenorio, R.D. González-Benítez,

M. Correyero-Plaza, J.C. Nieto, I. Janta, J.G. Ovalles-Bonilla, J. Martínez-Barrio,

B. Serrano, L. Valor, D. Hernández-Flórez, C.M. González, I. Monteagudo,

F.J. López-Longo. Rheumatology, Hospital General Universitario Gregorio

Marañon, Madrid, Spain

Background: Lung fibrosis (LF) is a type of interstitial disease that leads to lung scarring, respiratory failure and later on, death. There are 2 main types of LF: idiopathic and secondary; and the prognosis is very different. LF becomes relevant in connective tissue diseases (CTD) and some studies have suggested that there could be an association between anti-Ro/TRIM21 antibodies and the development of interstitial lung disease in these patients.

Objectives: The aim of this study was to assess if the presence of antiRo52/TRIM21 antibodies is an independent risk factor for developing CTDassociated LF. We also aimed to evaluate the initial manifestations of systemic diseases and clinical characteristics linked to certain antibodies.

Methods: It is a prospective, observational, longitudinal, single-center study conducted among unselected patients with CTD (rheumatoid arthritis, systemic lupus erythematosus (SLE), systemic sclerosis (SSc), polymyositis (PM), overlap CTD syndrome (OCTD), MCTD, primary Sjögren's sd (PSS), primary antiphospholipid $\mathrm{sd}$, systemic vasculitis, and undifferentiated connective tissue disease).

We analysed data from 1,432 caucasian patients included in the "Systemic Autoimmune Diseases (SAD) Registry" from 1988 to 2014. They were all checked at least biannually and blood samples were taken according to clinical practice. Exclusion criteria were LF as the initial manifestation of the SAD, LF already diagnosed at the first visit and also LF secondary to drugs or a specific environment.

Results: $10 \%$ of patients included in the study developed LF. The OR for LF in patients with anti-Ro52/TRIM21 antibodies was $1.757(95 \% \mathrm{Cl}=1.1-2.7)$. The OR for LF increased with every year of age $(O R=1.03,95 \% C l=1.02-1.04)$. Only 9 out of 146 patients with LF were positive for Anti-La/SS-B antibodies, and the OR for males was 8.6 compared to women $(95 \% \mathrm{Cl}=1.8-39.5)$. Patients with SSc and PM showed a higher OR for the development of LF $(6.995 \% \mathrm{Cl}=4.6-10.4$ and $2.0,95 \% \mathrm{Cl}=1.2-2.8$ respectively) compared to those diagnosed with other CTD. The time passed from the first symptoms to the diagnosis of LF was inversely related to the age of onset $(r=-.230 ; p=0.013)$. The average time was 60 months for patients with anti-Ro52/TRIM21 antibodies (29 patients) and 138 months for patients without them.

\begin{tabular}{|c|c|c|c|c|c|c|c|}
\hline & \multicolumn{4}{|c|}{ LF } & \multirow[t]{3}{*}{$\mathrm{p}$} & \multirow[t]{3}{*}{ OR } & \multirow[t]{3}{*}{ OR $95 \% \mathrm{Cl}$} \\
\hline & \multicolumn{2}{|c|}{ Yes $(n=146)$} & \multicolumn{2}{|c|}{ No $(n=1286)$} & & & \\
\hline & Mean $(\mathrm{Sd})$ & $n(\%)$ & Mean (Sd) & $n(\%)$ & & & \\
\hline anti-Ro/SS-A $60 \mathrm{kDa}$ & 39 & 26.7 & 307 & 23.9 & 0.47 & 1.16 & $0.78-1.71$ \\
\hline anti-Ro52/TRIM21 & 36 & 24.7 & 205 & 15.9 & 0.01 & 1.72 & $1.15-2.58$ \\
\hline anti-La & 9 & 6.2 & 129 & 10.1 & 0.14 & 0.58 & $0.29-1.17$ \\
\hline SLE & 25 & 17.1 & 398 & 30.9 & $<0.001$ & 0.46 & $0.29-0.72$ \\
\hline MCTD & 13 & 8.9 & 71 & 5.5 & 0.13 & 1.67 & $0.90-3.10$ \\
\hline OCTD & 13 & 8.9 & 52 & 4.0 & 0.01 & 2.32 & $1.23-4.37$ \\
\hline SSc & 52 & 35.6 & 107 & 8.3 & $<0.001$ & 6.09 & $4.11-9.02$ \\
\hline PM & 26 & 17.8 & 126 & 9.8 & 0.006 & 1.99 & $1.25-3.16$ \\
\hline SSc-PM & 8 & 5.5 & 16 & 1.2 & 0.002 & 4.60 & $1.93-10.9$ \\
\hline PSS & 8 & 5.5 & 100 & 7.8 & 0.408 & 0.68 & $0.32-1.44$ \\
\hline Female & 30 & 20.5 & 229 & 17.8 & 0.42 & 1.19 & $0.78-1.82$ \\
\hline Age (y.) & 65.25 & 14.5 & 58.3 & 17.2 & $<0.001$ & 1.02 & $1.01-1.03$ \\
\hline
\end{tabular}

Figure 1. Multiple logistic regression predictions (the black line represents the Figure 1. Multiple logisticre

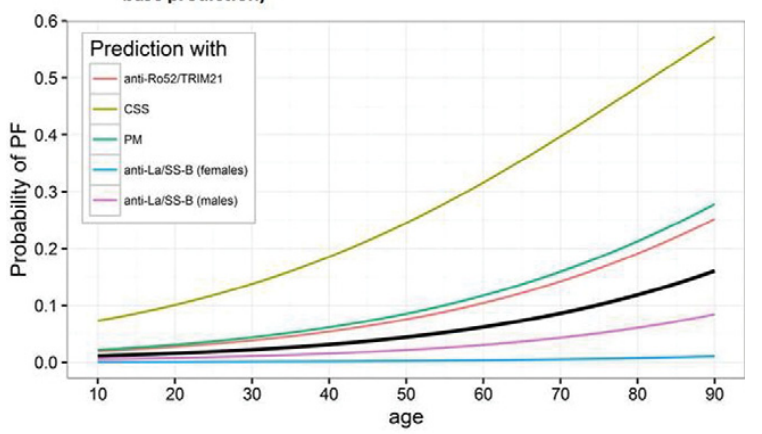

CSS: cutaneous systemic sclerosis; PM: polymyositis

Conclusions: Anti-Ro52/TRIM21 antibodies have been proved to be a risk factor for developing LF. The earlier the age of onset, the slower the progression to fibrosis. However, patients with anti-Ro52/TRIM21 antibodies tend to have a faster development, independent of the age of onset. Anti-La-SS-B antibodies seem to be a protective factor for the development of LF in both genders; but the association is stronger in women.

Disclosure of Interest: None declared

DOI: 10.1136/annrheumdis-2017-eular.2890 